\title{
Procesos de FORMACIÓN DEL REgistro ARQUEOLÓGICO, CONDICIONES AMBIENTALES Y USO DEL ESPACIO EN LA COSTA norte del golfo San Matías (Río Negro, Argentina)
}

\author{
Dalila V. Herrera Villegas ${ }^{\mathrm{a}}$ y Pamela G. Steffan ${ }^{\mathrm{b}}$
}

\begin{abstract}
Resumen
Entender la dinámica de los ambientes costeros es fundamental a la hora de dar cuenta de los procesos que allí han actuado a través del tiempo. Estos procesos son los que determinan las propiedades primarias de depositación de la evidencia cultural, asi como sus particularidades actuales. Es fundamental comprender e interpretar la historia de formación de los sitios arqueológicos, dado que estos van a permitir establecer la cronología y continuidad temporal del registro arqueológico. En este artículo se plantea la integración de resultados sedimentológicos, arqueomalacológicos y meteorológicos de las localidades de Babia Creek y Faro San Matías de la costa norte del golfo San Matias. Se infiere la dinámica ambiental de formación de las geoformas, sus características de migración y se analiza la relación de estos factores con la representación y conservación diferencial del registro arqueológico. Se presta especial atención a la estacionalidad de formación de los concheros y los patrones de variación de los registros meteorológicos históricos que permiten delinear tendencias de preservación selectivas. Esto es particularmente relevante para discutir el uso del espacio, ya que la dinámica eólica puede afectar las propiedades cualitativas, cuantitativas, resolución e integridad de los depósitos arqueológicos.
\end{abstract}

Palabras clave: geoarqueología, concheros, arqueomalacología, registros meteorológicos.

\author{
Abstract \\ ARCHAEOLOGICAL REGISTRY FORMATION PROCESSES ENVIRONMENTAL CONDITIONS AND \\ USE OF SPACE ON THE NORTH COAST OF THE GULF SAN MATÍAS (RÍO NEGRO, ARGENTINA)
}

Understanding the dynamics formation of coastal environments is essential when accounting for the processes that have taken place in the coast line over time. These processes are what determine the primary deposition properties of the cultural evidence, as well as its current characteristics. To establish the chronology and temporal continuity of the archaeological record, it is essential to understand and interpret the taphonomic history of archaeological sites. In this article we propose the integration of sedimentological, archeomalacological and meteorological results of Bahia Creek and Faro San Matias localities on the north coast of the San Matias gulf. The environmental dynamics of geoform formation and their migration characteristics are inferred and an analysis of the relationship between these factors, the representation and differential conservation of the archaeological record. We paid special attention to the seasonal changes in the shell middens formation and the variation patterns of historical meteorological records, which allow us to delineate selective preservation trends. This is particularly relevant to discuss the use of space, since wind dynamics can affect the qualitative, quantitative, resolution and integrity properties of the archaeology deposits.

Keywords: geoarchaeology, shell middens, archaeomalacology, meteorological records.

a https://orcid.org/0000-0001-8942-9185. INCUAPA-CONICET-UNICEN. dalila1083@gmail.com

b https://orcid.org/0000-0003-4300-9278. INCUAPA-CONICET-UNICEN. psteffan@soc.unicen.edu.ar

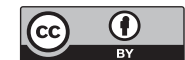




\section{Introducción}

La historia de formación de los sitios arqueológicos es determinante a la hora de dar cuenta del comportamiento humano en el pasado, ya que permite conocer los factores naturales y culturales que en ellos tuvieron lugar a través del tiempo. En la costa norte del golfo San Matías se reportan diferentes grupos morfológicos de dunas (principalmente parabólicas y barjanoides), con cronologías disímiles. Su génesis se halla vinculada con diferentes pulsos de descenso del nivel del mar ocurridos desde el máximo transgresivo del Holoceno Medio en la región (Schellmann y Radtke 2010). Esto es sustentado por trabajos paleoclimáticos previamente realizados en la costa norte patagónica (Schäbitz 2003). Con estos datos se pudo dar cuenta de la relación de estas variables con la preservación, visibilidad y disposición actual de la evidencia arqueológica (Favier Dubois y Borella 2007). Asimismo, fue posible establecer las probabilidades de que la duna conserve su morfología y que el registro arqueológico preserve sus propiedades de formación, ya que muchas de las características de la evidencia cultural están directamente relacionadas con la dinámica costera, específicamente con la sedimentaria.

En este trabajo nos enfocaremos en las dunas barjanoides sobre las que se encuentran emplazados los concheros de las localidades Paesani (Bahía Creek) y Faro San Matías con cronologías del Holoceno Tardío. En este artículo se propone analizar las variaciones de los agentes meteóricos (intensidad y dirección de los vientos de acuerdo con la estacionalidad) a partir de registros históricos y las particularidades geomorfológicas del área. Con estos resultados se discute la dinámica ambiental costera y sus implicancias en el uso y función de los espacios en dicha costa. La utilización de estos podría ser el resultado de la optimización de tiempo y energía en la obtención de los recursos; así como de la búsqueda de zonas de reparo debido a los fuertes vientos que azotan la región. Entre tanto, con esta información, se podría obtener además datos valiosos de la potencialidad de preservación de los sitios arqueológicos dado que muchas de sus propiedades se deben en gran parte a las condiciones ambientales preponderantes y a la presencia de espacios de rápida sedimentación. Teniendo en cuenta lo dicho, se propone como objetivo general dar cuenta de los procesos de formación naturales y culturales que actuaron y actúan sobre del registro arqueológico. Por ello se plantea integrar resultados de análisis sedimentológicos y arqueomalacológicos de ambas localidades mencionadas previamente, analizar los registros meteorológicos históricos y discutir su influencia en la dinámica geomorfológica y finalmente, se evalúa la utilidad de incluir registros históricos en estudios del pasado y sus limitaciones.

\section{2. Área de estudio}

La zona de estudio se extiende desde el balneario El Cóndor hasta la bahía de San Antonio, litoral que posee una dirección general este-oeste, (Fig. 1), caracterizándose por un clima templado semiárido, con temperaturas de unos $12{ }^{\circ} \mathrm{C}$ y precipitaciones medias entre 100 y 350 milímetros anuales (Labraga y Villalba 2009). Las mareas presentan un régimen semidiurno y una amplitud media de 3.20 metros en la desembocadura del río Negro, mientras que a la entrada de la bahía de San Antonio esta es de 7.10 metros (Capítoli 1973). Los vientos predominantes soplan del cuadrante oeste (noroeste, oeste y sudoeste) presentando mayor intensidad entre los meses de septiembre y enero, alcanzando velocidades históricas máximas las del cuadrante sudoeste (Crisoliti y Pahissa Campá 1973). En cuanto a la vegetación, domina la estepa arbustiva correspondiente a la provincia de monte, distrito sur (Cabrera y Willink 1980; Roig et al. 2009) predominando el tomillo, la jarilla y el molle, mientras que en algunos sectores se observa el algarrobo patagónico y algo de chañar (Bran et al. 1985). 

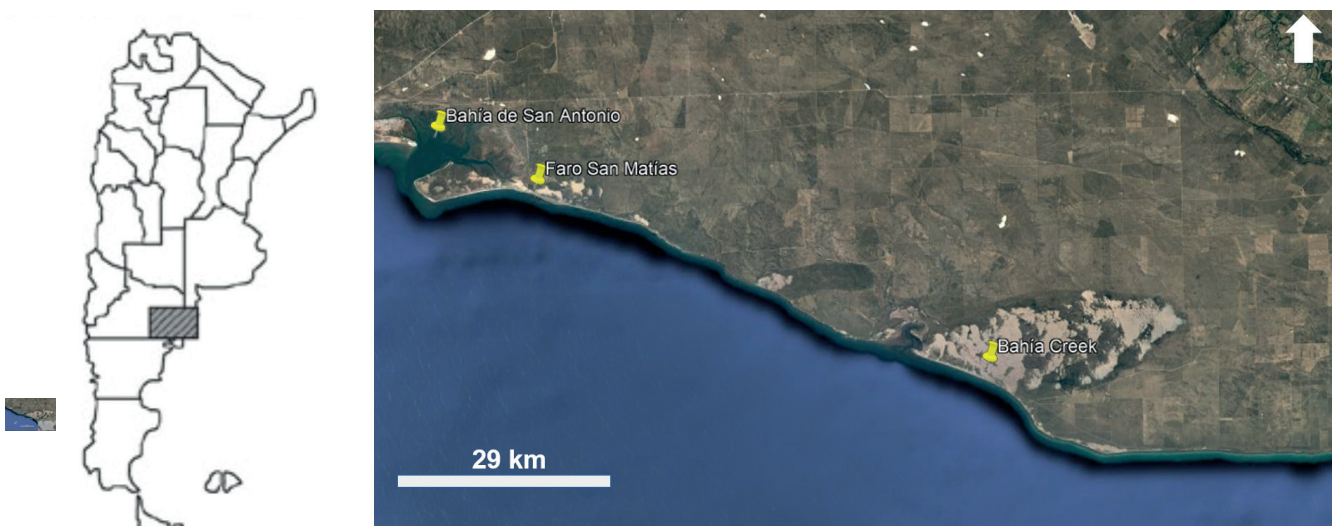

Figura 1. Área de estudio en la costa norte del golfo San Matías (Río Negro, Argentina). Ubicación de los sitios Faro San Matias y Paesani (Bahia Creek).

\subsection{Morfologías eólicas en la costa norte del golfo San Matías}

Una de las características más sobresalientes de la costa rionegrina es que en esta franja costera se reportan dos tipos de dunas (parabólicas y barjanoides) con cronologías disímiles producto de variaciones en la disponibilidad de arena en la playa en función de cambios del nivel del mar (Favier Dubois et al. 2016). Los estudios geológicos y geomorfológicos realizados en las proximidades de la región dan cuenta de condiciones áridas durante el máximo transgresivo del Holoceno medio ( $c a .7400$ ańos AP), período en el que las elevadas temperaturas globales provocaron un incremento del nivel del mar entre 6 y 7 metros por encima del nivel actual. Posteriormente, hacia el $6000 \mathrm{AP}$ se produce un primer pulso de descenso del nivel marino en la costa patagónica (Schellmann y Radtke 2010) al que le sigue una etapa de estabilidad hasta hace unos 3000-2600 ańos AP. Durante esta etapa cuando el aporte sedimentario desde la playa no es abundante debido al elevado nivel del mar, se empiezan a desarrollar lo que hoy observamos como campos de dunas parabólicas en la región. Luego, entre ca. 2600-2400 años AP se reporta un segundo pulso de descenso del nivel del mar no menor a 1 metro (Schellmann y Radtke 2010) que favoreció un mayor desarrollo de cuerpos eólicos en forma de grandes campos de dunas barjanoides, particularidad relacionada directamente con una mayor provisión y movilidad de arena, condición que ha valido para que estas dunas sean las más abundantes en toda la costa norte.

La localidad arqueológica Faro San Matías y Paesani (Bahía Creek) (Fig. 1) se encuentran emplazadas en un paisaje costero dominado por dunas (Favier Dubois y Scartascini 2012; Borella et al. 2016, entre otros). Estas últimas no solo son importantes por ser las más comunes en toda la costa, sino porque sobre ellas se encuentran emplazados los sitios arqueológicos (Fig. 2). Aunque en estas dos localidades los campos de dunas cuentan con diferentes dimensiones, lo que más ha llamado la atención es el importante rasgo geomorfológico conformado por un enorme campo de dunas que se desarrolla entre la Caleta de los Loros y Bahía Creek que se introduce 45 kilómetros tierra adentro con dirección suroeste-noreste (Fig. 2). Dicho cuerpo de dunas evolucionó en sentido E-O debido a la intensidad de los vientos que soplan del SO y al material sedimentario disponible en la playa para ser transportado por el viento (Favier Dubois y Borella 2011). La presencia de esta lengua arenosa está conformada principalmente por un grupo de dunas barjanoides, cuya morfología está relacionada con una mayor provisión y movilidad de arena producto del bajo nivel marino.

El área se caracteriza por la riqueza y la variedad de su registro arqueológico y bioarqueológico desde el Holoceno Medio y Tardío (Bórmida 1964; Gómez Otero 2007; Scartascini 2010). Las reiteradas ocupaciones humanas del Holoceno Tardío en la costa rionegrina (Tabla 1) 




Figura 2. Se destaca la presencia de dunas barjanoides y en el recuadro inferior derecho se muestra otra imagen en la se realza la morfología de la duna.

se registran en los concheros, donde las acumulaciones de valvas se encuentran en asociación a restos arqueofaunísticos (Borella et al. 2011). Se trata de estructuras de descarte de valvas formadas principalmente por mejillones (Mytilus platensis) y cholgas (Aulacomya ater) y en menor medida mejillines, crepídulas y restos óseos, con un espesor que no supera los 30 centímetros y una extensión variable en planta entre 1 a 10 metros de largo (Favier Dubois y Borella 2011). En general, los concheros de la localidad arqueológica Faro San Matías se localizan en dunas que sin dudas ofrecieron lugares reparados en el pasado (Favier Dubois et al. 2008; Borella et al. 2011). En la actualidad, la erosión reciente de las dunas deja expuestos los materiales arqueológicos y es posible observar los concheros en estratigrafía.

Tabla 1. Cronología de las ocupaciones humanas en la costa rionegrina.

\begin{tabular}{lcccl}
\hline Sitio arqueológico & $\begin{array}{c}\text { Material } \\
\text { datado }\end{array}$ & $\begin{array}{c}\text { Fecha radio- } \\
\text { carbónica } \\
\text { obtenida }\end{array}$ & LabRef. & Referencia \\
\hline FSM S2 & carbones & $2910 \pm 90$ & LP-1878 & Favier Dubois, Borella y Tykot 2009 \\
\hline FSM Mont. I & valvas & $1680 \pm 90$ & LP-2352 & Favier Dubois 2013 \\
\hline FSM Mont. II & valvas & $1630 \pm 70$ & LP-2358 & Favier Dubois 2013 \\
\hline FSM S6 & carbones & $1380 \pm 90$ & LP-1873 & Favier Dubois, Borella y Tykot 2009 \\
\hline B. Creek, Paesani S1 & valvas & $1100 \pm 90$ & AC-1710 & Favier Dubois y Borella 2007 \\
\hline
\end{tabular}


Figura 3. Item $A$ da cuenta de la importancia que tuvo la dinámica costera en el aporte sedimentario que dio paso al rápido sepultamiento de los concheros permitiendo que las dunas fueran estabilizadas por la vegetación permitiendo asi su preservación en el tiempo. Item B permite observar propiedades cualitativas y cuantitativas de las valvas, es decir que estas aún se conservan enteras y mantiene parte de su color, lo que sugiere un rápido enterramiento.

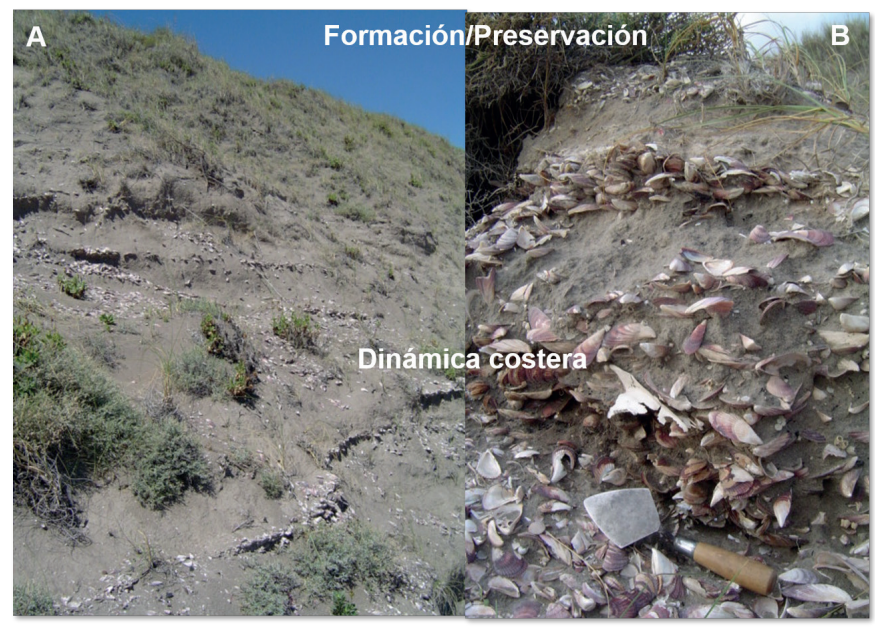

\section{Propiedades del registro arqueológico en las localidades de Faro San Matías y Paesani (Bahía} Creek)

Para poder dar cuenta del comportamiento actual de la evidencia arqueológica es necesario conocer los agentes que han tenido lugar sobre este a lo largo del tiempo. En este sentido, (Schiffer 1991, 1996, 2010) destaca dos procesos de formación. El primero tiene que ver con eventos culturales, y el segundo con los no culturales. Los procesos culturales son entendidos como aquellos que están relacionados con el comportamiento humano en el que un artefacto es modificado luego de un período inicial de uso o en una actividad específica, la cual se le conoce también como contexto sistémico. En cuanto a los procesos no culturales estos hacen referencia a aquellos eventos y procesos naturales que afectan los depósitos arqueológicos.

Los sitios arqueológicos presentes en las mencionadas localidades son el resultado de un comportamiento humano intencional de descarte, ya que esta evidencia responde a unas necesidades básicas de supervivencia. Esto puede ser entendido como un proceso cultural. En cuanto a los procesos naturales, se puede decir que estos tienen que ver con los efectos que el medio pueda llegar a tener sobre el registro arqueológico. En este sentido, cuando un sitio es abandonado, este queda expuesto a las condiciones medioambientales. En el caso específico, la dinámica costera permitió que las propiedades depositacionales de los materiales fueran preservados en el tiempo, ya que, gracias al aporte constante de material sedimentario proveniente de la playa hacia la duna a través del viento, hicieron que los sitios fueran sepultados rápidamente, impidiendo que estos fuesen destruidos (Favier Dubois y Borella 2007) (Fig. 3).

Debido al proceso de degradación que viene sufriendo este paisaje costero en las últimas décadas, han disminuido las probabilidades de que cualquier tipo de evidencia arqueológica sobreviva, es decir que el contexto sistémico original de estos sitios conserve gran parte de sus propiedades primarias de depositación. De acuerdo con esto, existen dos tipos de erosión; la primera de ellas obedece a cambios propios del ambiente, tales como; variaciones por sequías cortas o períodos más largos de aridez (McKee 1983; Waters 1992) variables que tienden a determinar la presencia o ausencia de vegetación, siendo esta de vital importancia en la cohesión y estabilidad de las dunas, evitando así que estas sean erosionadas y por ende reactivadas.

El segundo factor está dado por una serie de actividades antrópicas que para en el caso de la costa norte iniciaron su desarrollo en el siglo XX con la introducción de ganado ovino y vacuno en las últimas décadas, aspecto que propició la aparición de focos erosivos y modificación de la cubierta vegetal nativa, así como la aparición de actividades recreativas en las que el uso de vehículos $4 \times 4$ ha generado la fragmentación de material lítico, desintegración y compactación de las valvas 


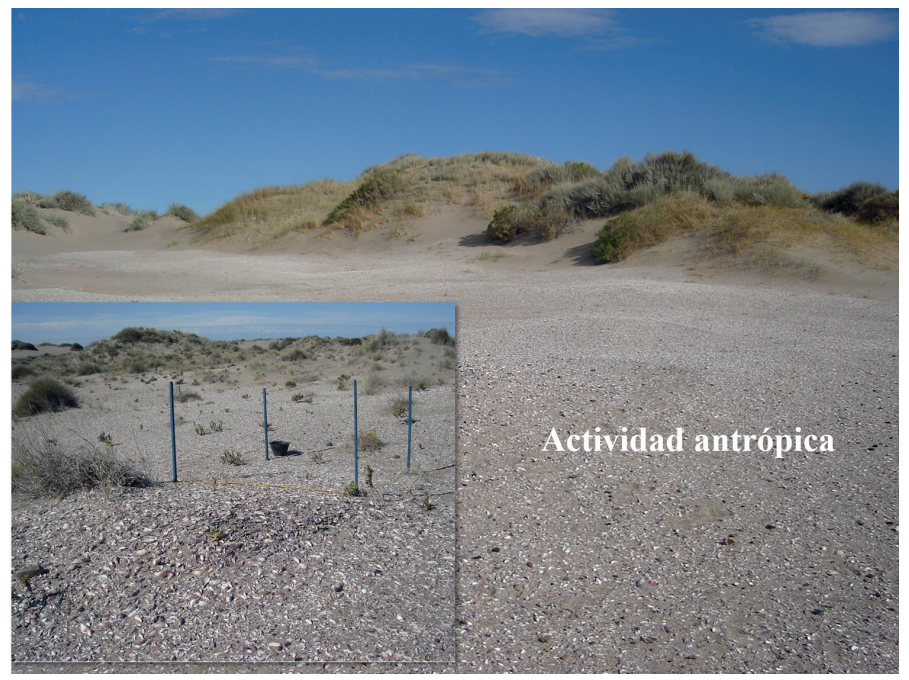

Figura 4. Se observa una gran mancha blanca que corresponde a fragmentos de valvas triturados $y$ desplazados a grandes distancias producto de la actividad antrópica actual.

expuestas (Favier Dubois y Borella 2007). Asimismo, este tipo de actividades ha venido acelerando la degradación de las dunas y la exposición de la evidencia arqueológica, condiciones que terminan afectado el contexto primario de depositación, ya que al perder su capa de vegetación los diferentes materiales empiezan a quedar expuestos y seguidamente removidos, desplazados y redepositados (Fig. 4) dificultando la individualización de los espacios. Aunque no se puede desconocer que ambos agentes han contribuido con la ubicación de estos sitios, también son los principales responsables de la afectación a la que se ve sometida la evidencia por su exposición a la intemperie, aspecto que claramente repercute sobre nuestro entendimiento acerca del contexto arqueológico.

Como consecuencia de la erosión a la que han sido sometidos los concheros fue posible identificar varios aspectos que tiene que ver con los procesos de formación que dieron origen a estas acumulaciones. Entre esos se pudo establecer que los concheros de la localidad FSM se encontraban ubicados sobre terrazas marinas holocenas y pleistocenas ubicadas a distancias variables de la playa actual. En estratigrafía se observó una concentración de valvas, con una geometría lenticular a tabular, intercalada con carbones, que presentaron un espesor de 40 centímetros, lo que podría apuntar a un solo evento de descarte (Favier Dubois et al. 2015), llegando así a corresponderse al hecho de que no se observaron diferencias que permitieran separar eventos depositacionales o discordancias internas. En consecuencia la cronología de cada conchero expresa un único evento de ocupación. En cuanto al arreglo interno de las valvas, estas no presentan un arreglo preferencial en el espacio y poseen una inclinación principalmente horizontal. Pero además de esto, se pudieron establecer alteraciones posdepositacionales tales como la presencia de armadillos y cuevas de roedores. El análisis de estas y otras variables condujo a pensar en que estos concheros son la respuesta a eventos de depositación primarios, discretos y de elevada resolución (Favier Dubois et al. 2016).

\section{Materiales y métodos}

La metodología aplicada en este trabajo consiste en realizar un acercamiento geoarqueológico a la zona de estudio. Para tener una mayor aproximación al proceso sedimentario en la localidad de Bahía Creek, principal campo de dunas en toda la costa norte de Patagonia, se llevó a cabo la recolección de muestras sedimentarias. Para esto, se determinó realizar una transecta de muestreo que abarcara diferentes unidades geomorfológicas que incluían la playa actual, terraza holocena, 




Figura 5. Detalle de la localización de la transecta de muestreo sedimentológico en el área.

dunas y conchero sobre duna (Fig. 5). Con estas muestras se buscaba realizar un análisis sedimentológico con el fin de evaluar variables texturales, como granulometría y selección, para de esta manera determinar el origen y dinámica sedimentaria entre las dunas y la playa. Para la determinación de la estructura de los granos y el predominio mineralógico de cada muestra se utilizaron 10 gramos de sedimentos. Para la identificación de la madurez textural y mineralógica se utilizó la propuesta de Corbí y Martínez (2015). El material fue fotografiado en microscopio estereoscópico marca LEICA S6D con un aumento de 6.3x. El criterio para tomar la foto de los sedimentos fue el tamaño de grano predominante de cada muestra. Los estudios granulométricos se realizaron utilizando la clasificación USA standard ASTM E 11-61. Para pesar las muestras se utilizó una balanza electrónica (A\&D FX 3000i). El proceso de tamizaje se realizó en varias etapas seleccionando distintas aberturas de malla (entre 1 y 0.074 micrones) hasta lograr retener el tamaño de grano correspondiente para cada tamaño granulométrico. Después se creó una tabla de Excel en la que se ingresó el peso retenido de cada muestra. Con estos datos se generó un histograma en el que fuera posible dar cuenta del porcentaje en peso de cada muestra recolectada de las unidades geomorfológicas previamente citadas.

El material arqueomalacológico proviene de los concheros de las localidades arqueológicas Faro San Matías y Paesani (Bahía Creek). Para este análisis se usó la especie de bivalvo Mytilus platensis ya que es la especie de mayor abundancia en los concheros. En el laboratorio del Instituto INCUAPA-CONICET, las valvas se limpiaron con un cepillado suave y se enjuagaron con abundante agua. Se dejó secar la muestra a temperatura ambiente. Posteriormente, se cuantificaron las valvas enteras de cada muestra.

La esclerocronología ha demostrado ser una herramienta útil para determinar la estacionalidad de muerte de los bivalvos (v.g. Oschmann 2009). Para identificar el patrón de su crecimiento, se aplican los resultados obtenidos en estudios previos del análisis macroscópico de valvas actuales colectadas en el área de estudio (Steffan y Morsan 2015). El registro del tipo de anillo de crecimiento (opaco o traslúcido) en el borde externo de valvas actuales indican un ciclo de depositación que alterna una banda opaca durante los meses más cálidos, entre octubre y marzo y una translúcida durante los meses invernales y principios de primavera (entre mayo y septiembre). El análisis esclerocronológico de las muestras arqueológicas se realiza a partir de la observación del borde externo de cada valva. Se registró a trasluz el tipo de anillo de crecimiento (opaco o traslúcido) 


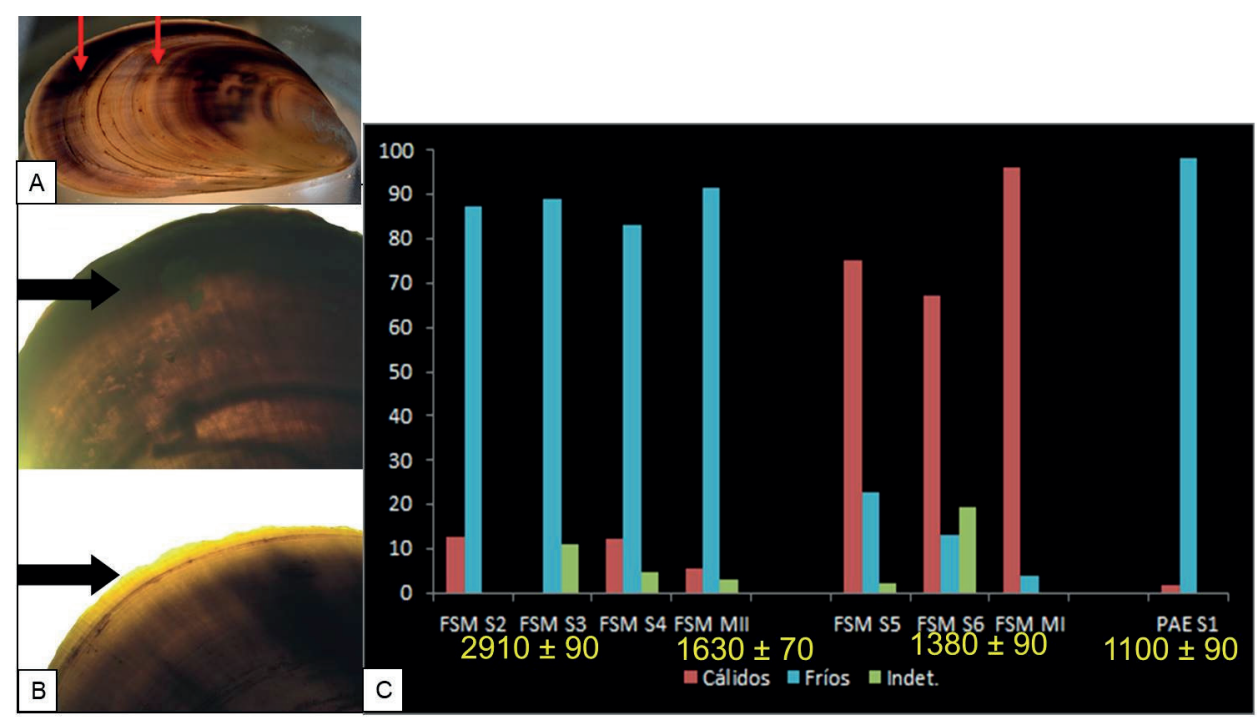

Figura 6. Análisis esclerocronológico de las valvas de Mytilus platensis. (A). A trasluz se observa la sucesión de anillo de crecimiento (opaco y traslúcido) de cada valva actual y su relación con la estacionalidad. (B). Se indica con una flecha el borde externo opaco (correspondiente a meses cálidos de crecimiento) y el borde translúcido (correspondiente a meses frios). (C). Las barras expresan el porcentaje (\%) d la uiltima banda opaca de crecimiento (opaco, trashúcido e indeterminado) identificada en el borde externo de las valvas arqueológicas provenientes de los sondeos de FSM y Babia Creek.

de cada valva, observado con luz transmitida en estéreo-microscopio (Fig. 6A). Los registros fueron contabilizados como porcentajes de cada tipo de banda en cada muestra. La prevalencia de uno y otro tipo permite inferir el momento de depositación en relación con la estación del año.

\subsection{Análisis de agentes meteorológicos}

Se llevó a cabo un relevamiento estadístico de datos meteorológicos provenientes de registros históricos de distintos rangos temporales a partir de diferentes fuentes de información. Se comparan tres períodos, entre los años 1941 y 1965 se utilizó la información compilada por el Instituto de Biología Marina de la provincia de Río Negro (Crisoliti y Pahissa Campá 1973) y los registros históricos de la estación meteorológica de San Antonio Oeste correspondientes a los períodos 1991-2000 y 2001-2010 (Servicio Meteorológico Nacional 2019). Las variables analizadas fueron: temperatura (valor promedio mensual, media anual, meses de valores mínimos), humedad relativa (valores mensuales), vientos (velocidad media mensual, velocidad media por dirección y frecuencia de dirección, valores máximos y mínimos por mes).

\section{Resultados}

\subsection{Resultados granulométricos}

Los resultados de los análisis granulométricos de las arenas de la playa de Faro San Matías arrojaron una variedad de tamaños, pero se evidenció un predominio de arenas finas (0.177 milímetros) (Fig. 7A). Si se comparan los resultados de la playa y la duna, se observa que la variedad de tamańos desaparece, aunque las arenas finas ( 0.177 milímetros) se mantienen, pero hay un predominio de arenas muy finas (Fig. 7B). En lo que respecta a la localidad de Paesani (Bahía Creek) los resultados no arrojan mayores diferencias. En esta, las arenas de la playa no presentan 

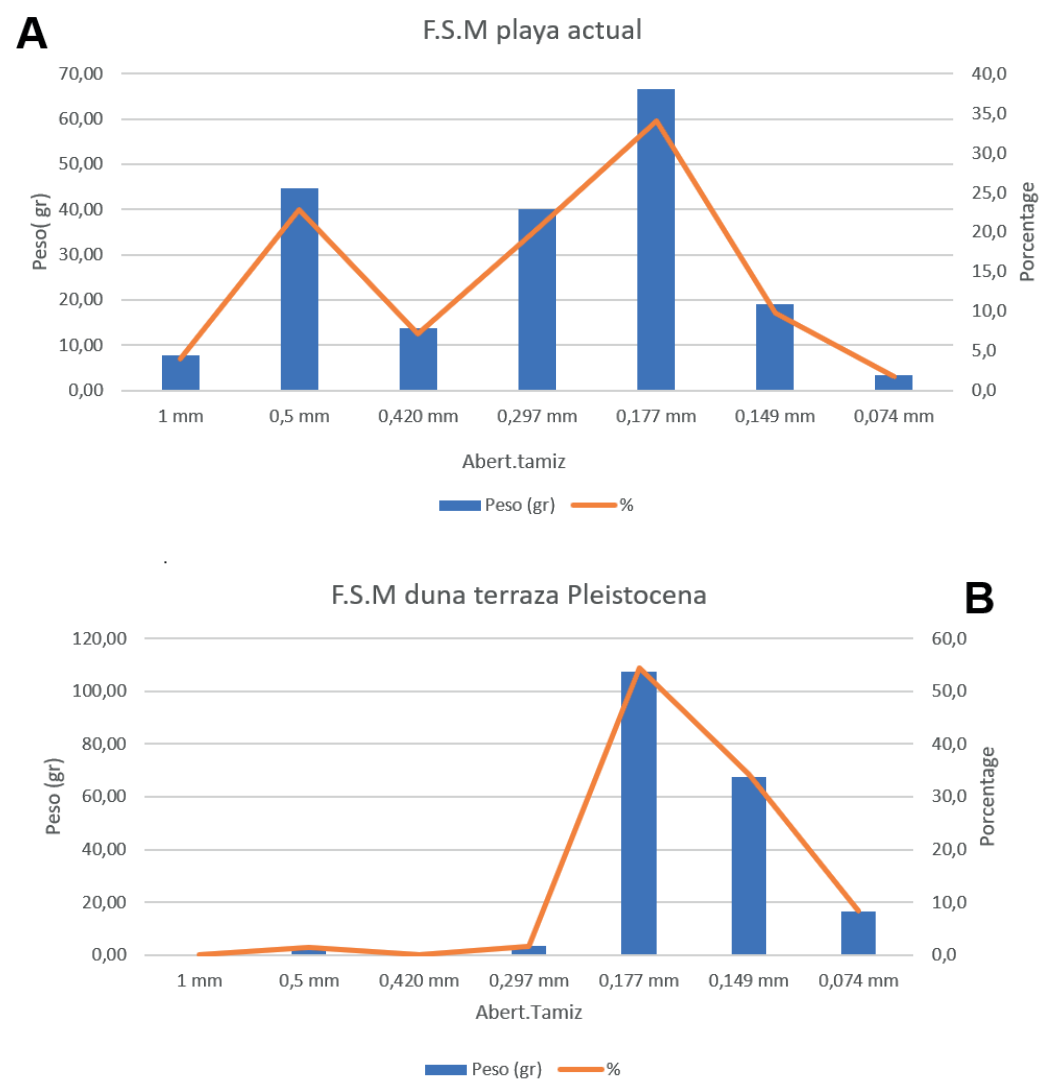

Figura 7. Análisis comparativo de los resultados granulométricos de la playa actual (A) y dunas (terraza pleistocénica) de Faro San Matías (B).

una variedad granulométrica marcada, pero sí una mayor presencia de arenas medias y finas (Fig. $8 \mathrm{~A})$. En cuanto a las arenas de la duna, se observa un predominio de arenas finas y muy finas, lo que se sería correspondiente con su origen eólico (Fig. 8B).

En cuanto a la madurez textural y mineralógica de las muestras recolectadas en las unidades geomorfológica de playa y duna de estas dos localidades, tampoco se pudo dar cuenta de modificaciones marcadas. Esto se debe a que en estas muestras se observó una gran variedad de minerales, lo que sería correspondiente a una baja madurez mineralógica. En términos texturales, las partículas no dieron cuenta de un predominio estructural, en tanto que, en todas las muestras los granos se encontraban entre subangulares y subredondeados (Fig. 9).

\subsection{Resultados arqueomalacológicos}

Los datos analizados en este trabajo se integran a los resultados obtenidos previamente (Favier Dubois et al. 2015). El análisis esclerocronológico de la totalidad de las valvas de Mytilus platensis recuperadas en los concheros de FSM permitió complementar las tendencias de la estacionalidad de colecta propuesta (Fig. 6B) en estudios previos (Borella et al. 2016). La muestra analizada se compone de 595 valvas provenientes de las localidades FSM y Bahía Creek. En FSM se analizaron siete sondeos: sondeo S2 ( $\mathrm{n}=48)$, S3 $(\mathrm{n}=27), \mathrm{S} 4(\mathrm{n}=107), \mathrm{S} 5(\mathrm{n}=128), \mathrm{S} 6(\mathrm{n}=46)$ y otros dos denominados Montículos (M) I ( $\mathrm{n}=54)$ y MII ( $\mathrm{n}=70)$ y en la localidad Paesani (Bahía Creek) se analizó el S1 ( $\mathrm{n}=115)$. 

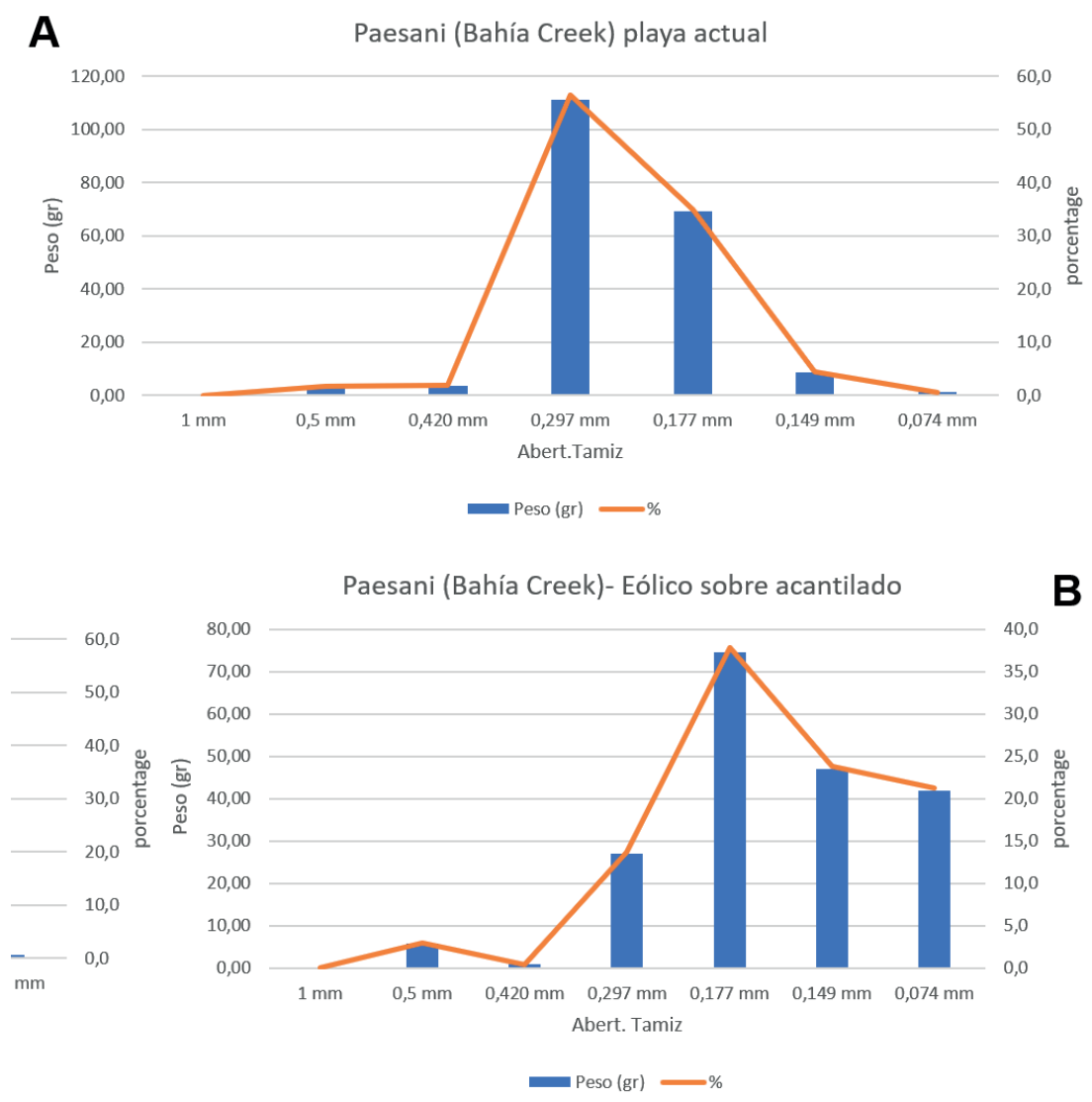

Figura 8. Análisis comparativo de los resultados granulométricos de la playa actual (A) y eólico del acantilado costero de Paesani (Bahia Creek) (B).

En los sondeos 5 y 6 (1380 años AP) el mayor porcentaje de la última banda de crecimiento opaca de las valvas (entre 67 y 90\%) indica que la colecta se habría llevado a cabo durante los meses cálidos, de octubre a marzo. Los resultados del sondeo Montículo II (91\% de bordes opacos) (1680 años AP) indican una estacionalidad similar (Fig. 6C). Las inferencias de estacionalidad generadas a partir de las valvas son coincidentes con la estacionalidad indicada a partir de la lectura de las bandas de crecimiento de un diente de Otaria flavescens (Borella et al. 2016).

El patrón de crecimiento de la mayor cantidad de valvas analizadas en el sondeo 2 ( $c a .2910$ años AP) (87\%), 3 (88\%), 4 (83\%), 12 (91.5\%) y el Montículo I (96\%) (ca. 1630 años AP) presentan la última banda de crecimiento traslúcida, la cual indica que la colecta corresponde un período de bajas temperaturas de invierno y principios de primavera, entre mayo y septiembre (Fig. 6C). La actividad de marisqueo en FSM se habría desarrollado en diferentes épocas del año.

No obstante, si tenemos en cuenta los diferentes eventos de ocupación y marisqueo durante el Holoceno Tardío, los fechados más tempranos en FSM corresponden a colectas realizadas durante los meses fríos. Los fechados desde 1600 años AP a $c a .1300$ años AP las colectas se realizaron en meses cálidos, excepto las valvas del sondeo Montículo II.

Las valvas Mytilus platensis analizadas en el sondeo 1 de Paesani indicaron que el mayor porcentaje $(98 \%)$ de la banda de crecimiento traslúcida corresponde a un período de colecta entre mayo y septiembre. Su cronología corresponde a la etapa final del Holoceno Tardío ( $c a$. 1100 ańos AP). 


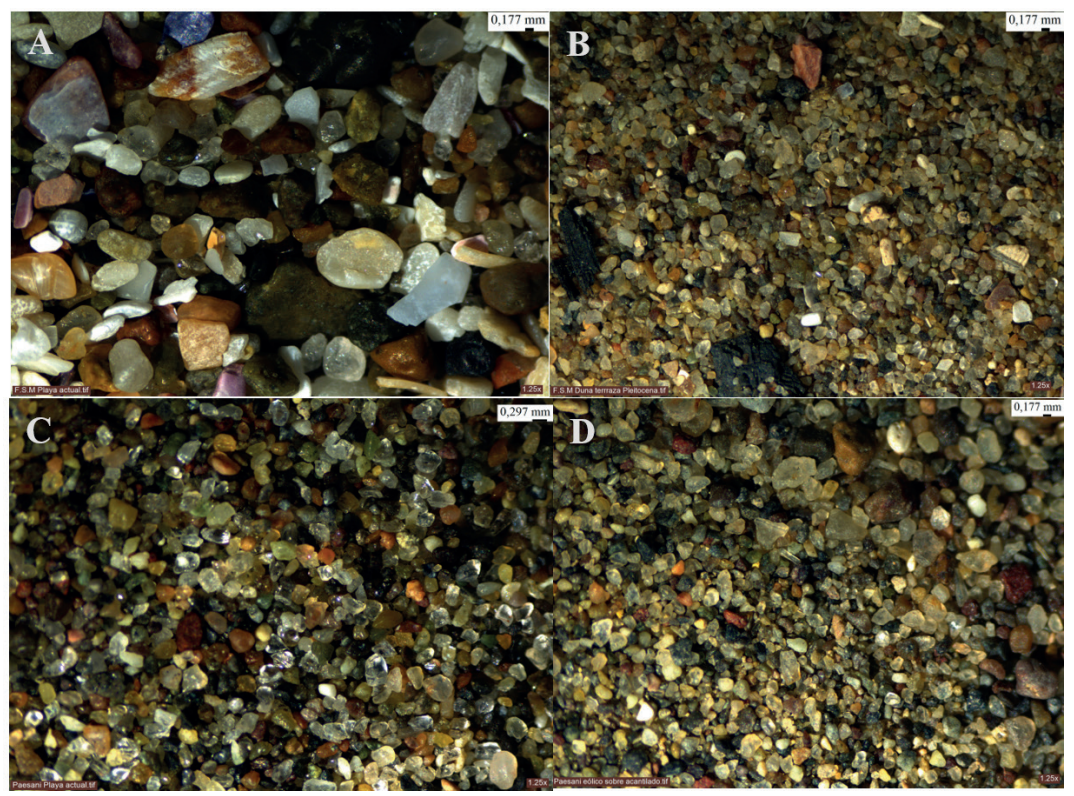

Figura 9. En las localidades de Faro San Matías (A y B) asi como de la localidad de Paesani (Babia Creek) (C y D). Se evidencia baja madurez textural, lo que significa que los granos de arena tienen una variedad de formas (redondeada, subredondeada, angular y subangular) lo que quiere decir que fueron poco transportado por el medio. Y la baja madurez mineralógica da cuenta de variedad de minerales en la que no predomina uno en especifico, esto también da cuenta del poco transporte que las arenas tuvieron hasta llegar a su depositación final.

\subsection{Relevamiento de registros históricos del Servicio Meteorológico Nacional}

Entre 1941-1965 la temperatura varió entre 22 y $7{ }^{\circ} \mathrm{C}$ reflejando una fluctuación estacional. A partir de los promedios de precipitación se estimó una línea de tendencia que refleja una mayor concentración de lluvias entre marzo y junio. Los vientos presentan su menor intensidad entre abril y mayo y a partir de junio registran su incremento.

Entre 1991-2010 la temperatura varió entre 22 y $6{ }^{\circ} \mathrm{C}$ reflejando una tendencia estacional similar al período histórico anterior. El promedio de precipitación señala una mayor concentración de lluvias entre marzo y mayo, descendiendo progresivamente hasta agosto, cuando incrementa nuevamente. Esta tendencia se diferencia del bloque temporal anterior, ya que el período de lluvias es más acotado. Los vientos presentan su mayor intensidad entre septiembre y enero y su menor intensidad entre marzo y mayo. A partir de junio registran un incremento, al igual que en el período anterior.

En cuanto a la dirección y velocidad de los vientos en los registros históricos de los períodos 1991-2000 y 2001-2010, estos indican que la dirección sur es preponderante durante los meses fríos y la mayor influencia del viento norte se registra durante los meses cálidos, coincidiendo con las tormentas de polvo y arena entre diciembre y marzo, mientras el viento del sudoeste presenta su máxima velocidad media entre noviembre y enero. En cuanto a la dirección del viento, para el período 1941-1965 no disponemos de datos cuantitativos; sin embargo, se destaca una preponderancia de los cuadrantes este, sudeste y sur durante enero. Se destaca que la mayor velocidad en $(\mathrm{Km} / \mathrm{h})$ del viento procede de la dirección sur a lo largo del ańo. Específicamente, para el período 2001-2010, observamos que la máxima velocidad del viento en casi todas las direcciones se registra en los meses más cálidos, excepto la dirección sur que registra su máxima velocidad durante los 


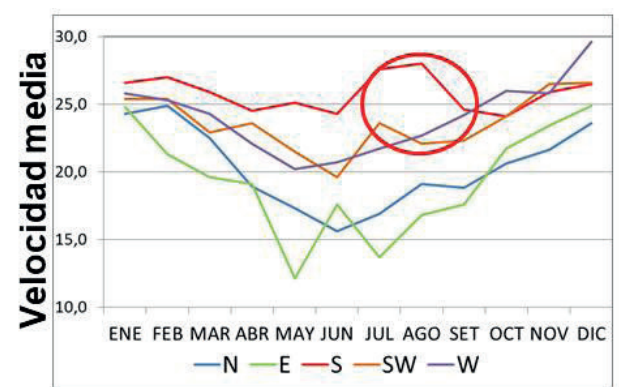

Dirección del viento en $\mathrm{Km} / \mathrm{h}$. $1991-2000$

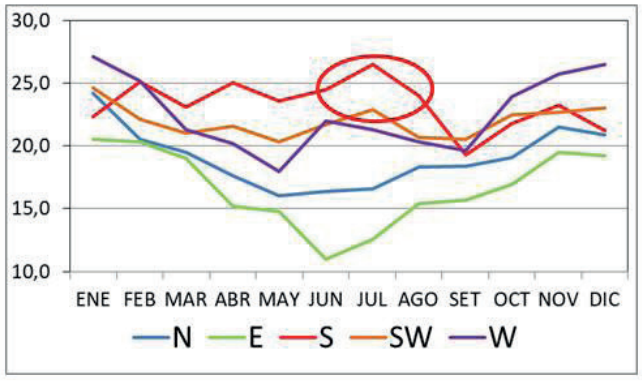

Dirección del viento en $\mathrm{Km} / \mathrm{h}$. 2001-2010

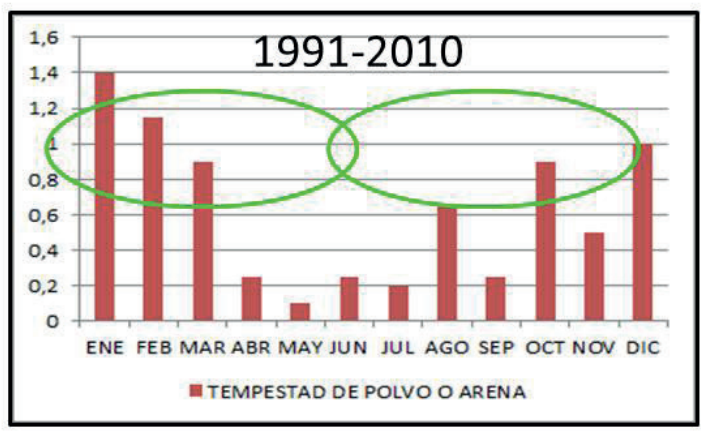

Figura 10. Registro de la dirección y frecuencia de los vientos del centro meteorológico nacional de la república de Argentina. Periodos 1991-2000 y 2001-2010.

meses fríos. Al igual que en el período anterior (1991-2000) la mayor velocidad proviene del sur a lo largo todo el año; sin embargo, se observan variaciones respecto del período anterior, ya que se registra mayor influencia del cuadrante oeste, en los meses más cálidos (entre octubre y febrero) (Fig. 10).

\section{Discusión}

Para poder entender las características actuales de la evidencia arqueológica en las localidades de Faro San Matías y Paesani (Bahía Creek), se requiere conocer y entender los procesos de formación que tuvieron lugar durante mucho tiempo en estas áreas, ya que estos son los responsables de muchas de las propiedades actuales del registro. Con base en los datos obtenidos del estudio comparativo de la composición sedimentaria entre la costa y las dunas sobre las que se encuentran emplazados los concheros de ambas localidades arqueológicas, fue posible delinear algunos procesos durante el Holoceno tardío. En este sentido, la variedad de tamaño granulométrico encontrada en las muestras analizadas de estas dos localidades, permite dar cuenta de la capacidad que tiene el viento para levantar y seleccionar el tamańo de los granos de arena, lo cual sugiere por un lado variaciones en la intensidad del viento y por el otro poco transporte, lo que es coincidente con la variedad mineralógica, es decir baja madurez textural de los sedimentos. Lo cual ha quedado registrado en estas morfologías eólicas. Este es un aspecto para destacar, dado el viento es sin duda uno de los agentes más importantes a la hora de transportar y depositar sedimentos. Con esta variedad queda claro lo determinante que es este agente para que se dé o no un rápido sepultamiento que favorezca la preservación de los depósitos. 
El contexto medioambiental en el que se encuentran emplazadas las localidades arqueológicas, ha sido afectado diferencialmente. Es por esto por lo que la propuesta teórico-metodológica deberá ajustarse en la medida de lo posible para responder las problemáticas propias de cada ambiente. En este sentido, cuando nos encontrarnos en un contexto cambiante como el costero, nuestro enfoque debe adaptarse de la mejor manera a cada variable que participa en la configuración actual del registro arqueológico, dado que es probable que este haya sufrido cambios en su disposición inicial a lo largo del tiempo. No obstante, una de las características de estos ambientes es que al ser tan dinámicos tienden a generar modificaciones sustanciales que han afectado estos espacios costeros a lo largo del tiempo, siendo estos el resultado de una serie de eventos que no solo lo han modificado, sino que han afectado la conformación primaria del registro arqueológico. Teniendo en cuenta la consideración de Waters y Kuehn (1996) de paisajes dinámicos y la relación con el grado de preservación de los sitios, se tienen en cuenta tanto las condiciones geomórficas de cada ambiente, como la duración, número e intensidad de los eventos de erosión y depositación de los mismos.

Los datos paleoambientales generados desde el registro polínico (Marcos et al. 2012), permiten obtener una señal regional de la disponibilidad de vegetación para el Holoceno Tardío en el área de estudio. El estudio mencionado registra ausencia de granos de polen de larga distancia como Nothofagus proveniente del sur entre 2900 y 2000 ańos AP. Es evidente que se produce un cambio de condiciones áridas a semiáridas con mayor influencia de la vegetación tipo espinal. En consecuencia, esto sugiere una menor influencia de los vientos del oeste o una menor intensidad de los mismos (Schäbitz 2003). Esta situación habría favorecido que las masas de aire húmedo del Atlántico aportaran mayores precipitaciones. A partir de 2000 años AP continúan las condiciones áridas a semiáridas, con menor disponibilidad de agua. Hacia los 1000 años AP, se registra un incremento de Nothofagus de larga distancia y un cambio más vinculado a la vegetación tipo monte. Entre 1000 y 500 años AP se infiere un pulso árido con la influencia de los vientos del oeste (Marcos et al. 2012). Los datos granulométricos presentados en este trabajo reflejan de manera coincidente las variaciones de la intensidad de los vientos del oeste que se registran desde el muestreo polínico para el área. Estos datos permiten inferir una mayor influencia de los vientos del oeste entre 2000 y 500 años AP.

La integración de los diferentes registros meteorológicos históricos explorados con la finalidad de conocer el comportamiento del viento en los últimos 50 ańos permite delinear tendencias similares entre los diferentes períodos. En el área de estudio la dirección preponderante del viento es del cuadrante sur durante la mayor parte del año. Sin embargo, en la intensidad (velocidad) y frecuencia del viento se observaron ciertas tendencias estacionales. En todos los registros fue posible dar cuenta que la mayor intensidad de vientos se registra en los meses cálidos (octubre y febrero) y la dirección es preponderantemente sur. Esto es coincidente con el período de registros de tempestad de arena y polvo. De esta manera se da cuenta de los períodos o estacionalidad en que los depósitos se vieron afectados debido a la intensidad de los vientos, pero que también pudieron favorecer el sepultamiento de unos y la exposición de otros.

Los estudios esclerocronológicos sobre Mytilus platensis llevados a cabo para ambas localidades permitieron definir la estacionalidad de formación de cada uno de los concheros vinculados a las actividades de marisqueo. La integración de los datos geoarqueológicos, malacológicos y meteorológicos presentada hasta el momento ofrece un marco de información interesante para discutir aspectos relevantes de preservación del registro y el potencial uso de estos sectores en el pasado.

Los datos meteorológicos históricos permitieron generar expectativas de preservación potencial de acuerdo con la estacionalidad de formación de los concheros. La preservación estaría influenciada por los vientos a lo largo del año. En los meses cálidos (noviembre-enero) se intensifica la influencia del sudoeste. Entre septiembre y enero el viento del norte genera eventos de tormenta de polvo y arena que habrían favorecido el sepultamiento de los restos arqueológicos. De acuerdo con la información meteorológica y estacional, consideramos que los concheros que se formaron 
durante los meses cálidos en comparación con las otras estacionalidades contaron con mayores y mejores probabilidades de preservación debido a un rápido enterramiento. No obstante, durante los meses fríos (mayo-septiembre) la mayor influencia del viento del sur y sudoeste, implican un mayor transporte de arenas, favoreciendo el enterramiento de los concheros. Otro aspecto potencialmente favorable para la preservación es la intensidad de los vientos, menor a $43 \mathrm{~km} / \mathrm{h}$, por lo cual la dispersión de los materiales por este agente habría sido leve e insuficiente para desplazar los materiales contenidos en la estructura antrópica (valvas, carbones y otros restos). Las condiciones ambientales y la presencia de espacios de rápida sedimentación permiten comprender los procesos de depositación de los concheros y la variada estacionalidad de colecta de valvas y otros recursos marinos registrados teniendo en cuenta la potencialidad de preservación de los sitios arqueológicos.

En lo que respecta a la preservación de los depósitos, creemos que al estar ubicados a favor del viento (cara a sotaventos) permitió que la preservación de los concheros fuera altamente positiva, ya que al encontrarse en esta zona de reparo facilitó que la duna al ir migrando fuese sepultando los concheros, aumentando así las posibilidades de que las propiedades depositacionales del material que conformaba el registro se preservara en buenas condiciones. Pero hay que destacar que esto se dio cuando la duna fue estabilizada por la vegetación, lo que permitió la preservación de los depósitos a través del tiempo, brindando así información valiosa en cuanto a los procesos que allí tuvieron lugar. Dentro de esos procesos se pudo determinar la importancia que tiene el viento para levantar y transportar sedimentos de la playa a la duna, facilitando el rápido sepultamiento de los depósitos y por ende la preservación de gran parte de sus propiedades primarias de depositación. De igual modo se pudo establecer el rol preponderante de la actividad antrópica en las propiedades depositacionales y posdepositacionales del registro arqueológico.

En consecuencia, las condiciones ambientales favorecieron la preservación de las propiedades cualitativas y cuantitativas del registro arqueológico presente en las localidades previamente mencionadas. Debido a esto, se pudieron obtener diferentes temporalidades que dan cuenta del uso de este espacio costero por parte de los grupos cazadores-recolectores. Esto permite considerar, que las dunas son las principales unidades portadoras del registro arqueológico.

La integración de nuevos datos apoya la propuesta planteada anteriormente (Favier Dubois y Borella 2007, 2011), de que las dunas fueron utilizadas como zonas de reparo debido a los fuertes vientos que azotan la región. El emplazamiento de los concheros en dirección a la cara de sotavento (a favor del viento) permite pensar que estos fueron espacios de reparo, ya que debido a que estas dunas pueden llegar a alcanzar hasta 100 metros de altura. No solo funcionaron como zonas óptimas de resguardo de los fuertes vientos que azotan la región, sino que además brindaría beneficios a la hora de mantener vivo el fuego, evidenciado por la abundante presencia de carbones en los cocheros, necesario para el tratamiento térmico de los moluscos, así como para la generación de calor. Esto permite entonces sugerir una alta funcionalidad y un uso recurrente de estos espacios (Fig. 11).

Para finalizar, se evalúa que los registros meteorológicos históricos y el patrón observado a partir de su relevamiento han sido significativamente valioso al momento de discutir no solo la dinámica geomorfológica costera, sino sus implicancias en el uso y función de los espacios en dicha costa. Con la integración y análisis de los datos expuestos fue posible dar cuenta de los agentes que participaron en los procesos de formación y transformación del registro arqueológico en las dos localidades estudiadas, y en cómo estos han modificado no solo las propiedades primarias de depositación sino la interpretación del contexto arqueológico. Bien sabemos que para poder entender los diferentes ambientes en los que se desenvolvieron las poblaciones pasadas y el registro que estas dejaron es fundamental dar cuenta de los agentes naturales y culturales que afectaron la evidencia material. Con base a estos resultados, hemos elaborado una propuesta de la distribución potencialmente probable de las dunas de acuerdo con la estacionalidad de formación de los concheros. Sin embargo, estas aproximaciones podrán ser testeadas desde otras líneas de investigación en el área. 


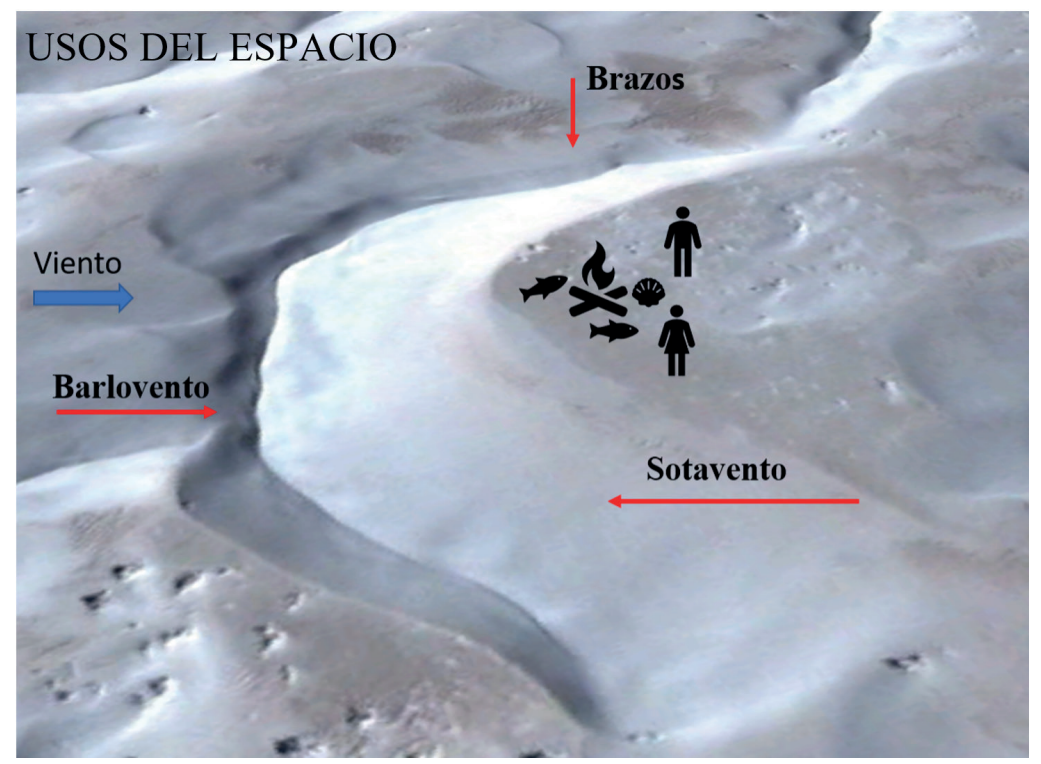

Figura 11. Esquema propuesto del uso del espacio en las localidades arqueológicas analizadas de la costa norte del golfo San Matias.

\section{Conclusiones}

Los estudios de la historia de formación y transformación del registro arqueológico en la dinámica geoambiental costera permiten evaluar la compleja interacción de diversos agentes que intervienen en su preservación. En consecuencia, el análisis de los procesos de formación permite comprender las variables que afectaron las propiedades primarias de sus aspectos espaciales y temporales. Su consideración permitirá una interpretación más parsimoniosa en cuanto al comportamiento de las poblaciones del pasado.

Los resultados sedimentológicos, esclerocronológicos sobre valvas y de los registros meteorológicos de las localidades Faro San Matías y Bahía Creek permiten comprender que la preservación del registro arqueológico ha estado influenciada principalmente por las características propias del ambiente dunar. Las variaciones estacionales de los vientos actuales nos permitieron inferir la dinámica eólica que ha sido un aspecto fundamental en el sepultamiento, preservación y exposición de la evidencia arqueológica, brindándole sus propiedades actuales.

La utilización de los registros meteorológicos históricos permitió generar expectativas de preservación potencial, vinculadas a la estacionalidad de formación de los concheros. El registro histórico y actual indican que la influencia de los vientos del oeste y sudeste influyeron en el transporte sedimentario que actuaron positivamente en la preservación de los concheros, lo cual contribuyó con la configuración espacial de los sitios arqueológicos.

A partir de este estudio, es posible considerar que la estacionalidad de colecta inferida desde el material arqueomalacológico es una muestra representativa de las actividades de marisqueo del pasado, ya que la dinámica estacional de los vientos que interviene en la formación y desplazamientos de las dunas habría favorecido el rápido enterramiento y preservación de los concheros. Teniendo en cuenta la dinámica eólica discutida en este trabajo, al parecer la mayor influencia de los vientos del oeste entre los 2000 y 500 años AP (evidenciada a través de estudios polínicos) podría haber favorecido la preservación de los eventos de ocupaciones humanas en Faro San Matías y Bahía Creek durante este lapso temporal. La integración de los datos ya expuestos, permitieron entender no solo la distribución actual de la evidencia arqueológica, sino el criterio de selección 
de los grupos humanos que ocuparon estos lugares en relación con el uso y función de las dunas en los ambientes costeros de la Bahía San Antonio.

\section{Agradecimientos}

A las/os organizadoras/es del VII GEGAL, a la Secretaría de Cultura y la Secretaría de Ambiente y Desarrollo Sustentable de la Provincia de Río Negro. Al Servicio Meteorológico Nacional, al PICT 2013-1128 y PICT 3575-2017 de ANPCyT, al INCUAPA- CONICET, a los doctores Cristian Favier Dubois y Florencia Borella por sus comentarios y sugerencias.

\section{Referencias}

Borella, F., F. Scartascini y H. A. Marani (2011). Explorando la subsistencia humana a partir de la evidencia faunística en la costa rionegrina, en: F. Borella y M. Cardillo (comps.), Arqueología de pescadores y marisqueadores en Nordpatagonia. Descifrando un registro de más de 6000 años, 87-110, Dunken, Buenos Aires.

Borella, F., M. Cardillo, F.L. Scartascini, P.G. Steffan, E. Carranza y C.M. Favier Dubois (2016). Faro San Matías 50 años después: Ocupaciones humanas durante el Holoceno Tardío (Nordpatagonia, Argentina), Revista de Antropología del Museo de Entre Ríos 2 (1), 62-76.

Bórmida, M. (1964). Arqueología de la costa norpatagónica, Trabajos de Prehistoria 14, 7-108.

Bran, D., G. Cecchi, A. Balmaceda y B. Lores (1985). Vegetación, en: Relevamiento Integrado de los Recursos Naturales de la Provincia de Río Negro, Convenio INTA- Ministerio de Recursos Naturales (Provincia de Río Negro), inédito, disponible en: Consejo de Ecología y Medio Ambiente (CODEMA), Viedma.

Cabrera, A. L. y A. Willink (1980). Biogeografía de América Latina, OEA Monographs, Washington, D.C.

Capítoli, R. (1973). El golfo San Matías en el ámbito del mar argentino, en: Relevamiento Ecológico y tipificación de las comunidades del Litoral Maritimo de la Provincia de Rio Negro, con especial referencia al establecimiento de áreas de cultivo para especies de interés comercial. Instituto de Biología Marina, 1-10, Asesoría de Desarrollo de Río Negro, Consejo Federal de Inversiones. Director del programa: S. R. Olivier, inédito, disponible en: Biblioteca del Consejo Federal de Inversiones (Capital Federal).

Corbí, H. y J. Martínez (2015). Interpretando ambientes sedimentarios: taller de sedimentología con arenas como actividad didáctica de Ciencias de la Tierra, Enseñanza de las Ciencias de la Tierra 23(2), 242-252.

Crisoliti L.E. y M.E. Pahissa Campá (1973). Análisis climáticos de las estaciones meteorológicas Patagones, San Antonio Oeste y Trelew, en: Relevamiento Ecológico y tipificación de las comunidades del Litoral Maritimo de la Provincia de Río Negro, con especial referencia al establecimiento de áreas de cultivo para especies de interés comercial, 13-88, Instituto de Biología Marina, Asesoría de desarrollo de Río Negro. Consejo Federal de Inversiones. Director del programa: S. R. Olivier, Inédito, Disponible en: Biblioteca del Consejo Federal de Inversiones (Capital Federal).

Favier Dubois, C. (2013). Hacia una cronología del uso del espacio en la costa norte del golfo San Matías (Río Negro, Argentina): sesgos geológicos e indicadores temporales, en: Zangrando, A., R. Barberena, A. Gil, G. Neme, M. Giardina, L. Luna, C. Otaola, S. Paulides, L. Salgán y Á. Tívoli (comps.), Tendencias teórico-metodológicas y casos de estudio en la arqueología de la Patagonia, 87-96. Buenos Aires.

Favier Dubois, C. y F. Borella (2007). Consideraciones acerca de los procesos de formación de concheros de la costa norte del golfo San Matías, Río Negro, Cazadores Recolectores del Cono Sur 2, 151-165.

Favier Dubois, C.M., F. Borella, L. Manzi, M. Cardillo, S. Lanzelotti, F. Scartascini, C. Mariano y E. Borges Vaz (2008). Aproximación regional al registro arqueológico de la costa rionegrina, en: I. Cruz y S. Caracotche (eds.), Arqueología de la Costa Patagónica, perspectivas para la conservación, 50-68, Universidad Nacional de la Patagonia Austral, Río Gallegos.

Favier Dubois, C., Borella, F. y R. Tykot (2009). Explorando tendencias en el uso humano del espacio y los recursos en el litoral rionegrino (Argentina) durante el Holoceno medio y tardío, en: M. Salemme, F. Santiago, M. Alvarez, E. Piana, M. Vázquez y E. Mansur (eds.), Arqueología de la Patagonia - Una mirada desde el último confin, 985- 997, Editorial Utopías, Ushuaia.

Favier Dubois, C. y F. Borella (2011). Contrastes en la costa del golfo: una aproximación al estudio del uso humano del litoral rionegrino en el pasado, en: F. Borella y M. Cardillo (eds.), Arqueología de pescadores y marisqueadores en Nordpatagonia. Descifrando un registro de más de 6000 años, 13-42, Dunken, Buenos Aires, 
Favier Dubois, C.M. y F. Scartascini (2012). Intensive fishery scenarios on the North Patagonian coast (Río Negro, Argentina) during the Mid-Holocene, Quaternary International 256 (1), 62-70. https://doi.org/10.1016/j. quaint.2011.07.041

Favier Dubois, C., Borella, F., Steffan, P. y F. Ortega (2015). Aportes al estudio de la contemporaneidad en las acumulaciones de valvas de origen antrópico en ambientes litorales, en: Cuadernos del Instituto Nacional de Antropologia y Pensamiento Latinoamericano - Series Especiales 2(4), 1-12.

Favier Dubois, C., Kokot R., Scartascini, F. y F. Borella (2016). Una perspectiva geoarqueológica del registro de ocupaciones humanas en el golfo San Matías (Río Negro, Argentina), La geoarqueología en la Argentina: Aportes y avances. Volumen especial, Intersecciones en Antropologia 4, 47-59.

Gómez Otero, J. (2007). Dieta, uso del espacio y evolución en poblaciones cazadoras-recolectoras de la costa centro-septentrional de Patagonia durante el Holoceno medio y tardío, tesis de doctorado inédita, Facultad de Filosofía y Letras, Universidad de Buenos Aires.

Labraga, J.C. y R. Villalba (2009). Climate in the Monte Desert: Past trends, present conditions, and future projections, Journal of Arid Environments 73(2), 154-163. https://doi.org/10.1016/j.jaridenv.2008.03.016

Marcos, M.A., M. V. Mancini y C.M. Favier Dubois (2014). Middle to late-Holocene environmental changes in Bajo de la Quinta, NE Patagonia, inferred by palynological records and their relation to human occupation, The Holocene 22(11), 1271- 1281. https://doi.org/10.1177/0959683612437862

McKee, E.D. (1983). Eolian sand bodies of the world, en: M.E. Brookfield y T.S. Ahlbrandt (eds.), Eolian sediments and processes, 1-26, Elsevier, Amsterdam.

Oschmann, W. (2009). Sclerochronology: editorial, International Journal of Earth Sciences 98, 1-2.

Roig, F.A., Roig-Juñent, S. y V. Corbalán (2009). Biogeography of the Monte desert, Journal of Arid Environments 73(2), 164-172. https://doi.org/10.1016/j.jaridenv.2008.07.016

Scartascini, F. (2010). Explotación de peces en la costa Norte del golfo San Matías (Río Negro): Cambios y continuidades en la Subsistencia y uso del espacio costero, tesis de pregrado, Facultad de Filosofía y letras, Universidad de Buenos Aires.

Schäbitz, F. (2003). Estudios polínicos del Cuaternario en las regiones áridas del sur de Argentina, Revista del Museo Argentino de Ciencias Naturales 5(2), 291-299.

Schellmann, G. y U. Radtke (2010). Timing and magnitude of Holocene sea-level changes along the middle and south Patagonian Atlantic coast derived from beach ridge systems, litoral terraces and valley-mouth terraces, Earth-Science Reviews 103(1), 1-30. https://doi.org/10.1016/j.earscirev.2010.06.003

Schiffer, M. (1991). Los procesos de formación del registro arqueológico, Boletín de Antropología Americana 23, $39-45$.

Schiffer, M. (1996). Formation processes of the archaeological record, University of Utah Press, Salt Lake City.

Schiffer, M. (2010). Behavioral archaeology: Principles and practice, Equinox Publishing Ltd., London.

Steffan, P. y E. Morsan (2015). Un abordaje metodológico para el estudio de estacionalidad de captura de Mytilus platensis (d'Orbigny, 1842) en la costa norte del golfo San Matías, en: H. Hammond y M. A. Zubimendi (eds), Arqueomalacología: abordajes metodológicos y casos de estudio en el Cono Sur, 108-116, Fundación de Historia Natural Félix de Azara, Ciudad Autónoma de Buenos Aires.

Waters, M.R. (1992). Principles of geoarchaeology: A North American perspective, The University of Arizona Press, Tucson.

Waters, M.R. y D.D. Kuehn (1996). The geoarchaeology of place: the effect of geological processes on the preservation and interpretation of the archaeological record, American Antiquity 61(3), 483-497. https://doi. org/10.2307/281836

Recibido: 22/06/2020

Aprobado: 14/10/2020 